\title{
Enhancing an Introductory Biomedical Engineering Course Using an Integrated Lecture-Lab Approach
}

\author{
Daniel P. Cavanagh, Samantha J. Richerson \\ Biomedical Engineering Program \& Department of Chemical Engineering / \\ Biomedical Engineering Program \& Department of Electrical Engineering \\ Bucknell University, Lewisburg, PA
}

Abstract

Over the past four years, the Introduction to Biomedical Engineering course at Bucknell University for non-majors has had a concomitant lab for the hands-on introduction of fundamental engineering concepts to the students. With the arrival of the first class of biomedical engineering majors into their introductory course, we have implemented a new teaching approach which is centered on the integration of lecture and lab. The underlying motivation of this new approach is to enhance retention of the fundamental concepts by students through the concurrent offering of lecture and hands-on laboratory experiences. We believe that this methodology has several benefits over traditional course formats where lecture and associated labs may be presented days apart. These benefits might include the ability to quickly address student misunderstandings which may arise during lecture, immediately reinforce complex technical concepts through hands-on activities and to provide the students with a real world application of the concepts.

To implement this course, we have taken advantage of the small class size (13 majors) and the recent opening of a new biomedical engineering hybrid instructional facility. This facility includes wet bench capabilities, lecture facilities, a range of flow, pressure, temperature and mass transport equipment, wireless laptops, physiological data acquisition systems, and electronic instrumentation clusters. With this facility, we have the ability to directly integrate lecture and valuable hands-on activities. For example, in the area of biotransport, a range of integrated sessions have been created to reinforce the learning of both simple and complex concepts. For the introduction of fluid properties such as viscosity, students are able to form groups and utilize viscometers during the class session to explore the range of viscous behaviors without leaving the room. For concepts such as unsteady mass balances, students might spend the first half of a class session working in groups on a theoretical model of a recirculating drug infusion problem. Immediately following the solution of the problem, students then move to the wet benches to run an experiment which demonstrates the theoretical result just derived.

Overall, we anticipate that this approach to integrating lecture and lab exercises will enhance the educational experience of the biomedical engineers. As the Biomedical Engineering 
Program incorporates direct assessment measures into the curriculum, the exact effects of this new teaching approach will ideally be identified.

Introduction

In fall 2004, Bucknell's first class of biomedical engineering majors, i.e., the Class of 2007, enrolled in our Introduction to Biomedical Engineering course at the beginning of their second year. Up until 2004, this introductory course was taught in a traditional lecture and lab fashion to students majoring in other engineering and science disciplines. As the size of the new biomedical engineering program is targeted to be approximately 60 students over all four years, we chose to identify a new teaching method for this course that is appropriate for this size class and that would enhance the effectiveness of the course. Furthermore, in planning our new engineering building which opened in fall 2004, we chose to create dedicated biomedical engineering instructional facilities that would permit the blending of traditional lecture and laboratory activities into single instructional sessions.

In taking advantage of the small class size and the new instructional facilities, we revamped our Introduction to Biomedical Engineering course for the majors to offer a course in which the boundaries between lecture and lab became less distinct and in which the students gained immediate reinforcement of technical concepts through a range of hands-on experimental, simulation and design exercises. Our goal was to create the opportunity for the faculty to be able to quickly address student misunderstandings which may arise during lecture, immediately reinforce complex technical concepts through hands-on activities and to provide the students with a real world application of the concepts. In the only other paper we are aware of which documents this style of instruction, faculty in the Department of Technology at the State University College at Buffalo designed a facility which permits the integration of lecture and lab for the purposes of exposing students to the application of engineering principles rather than the derivation and origin (Beasley, Culkowski and Guffner, 1990). From their experiences, they recognized that this technique enhances student-instructor interaction and the level of student accomplishment while also limiting student enrollment to 35 per facility and requiring more faculty contact time. As the primary mission of Bucknell is centered on undergraduate education and as our biomedical engineering program has been purposely design to provide a small number of students with an intense and interactive education, we feel that select courses in our curriculum are ideally suited for this instructional method. The current use of this method in our Introduction to Biomedical Engineering course is our first implementation of this instructional method.

\section{Course Description}

Introduction to Biomedical Engineering is a first semester, sophomore year course which is required of all biomedical engineering majors. In enrolling in the course, students are expected to have had at least two semesters of calculus, two semesters of calculus based physics and one semester of inorganic chemistry. A selection of general objectives of the course is listed below:

1. To introduce students to basic human physiology from the cellular to systemic levels. 
2. To expose students to the areas of biofluid mechanics, biological mass transport, bioinstrumentation and biomechanics.

3. To reinforce technical concepts with hands-on exercises.

4. To allow students to utilize current engineering and biomedical equipment to follow predetermined experimental protocols as well as to solve open-ended problems.

5. To present relevant applications of calculus to biomedical problems.

6. To encourage the students to identify and solve open-ended engineering problems following an engineering design process.

7. To introduce students to comparing and explaining the differences between experimental and theoretical results.

8. To show students the importance of multidisciplinary problems in biomedical engineering.

9. To require the students to utilize skills gained through analytical and experimental exercises to conduct a semester long group design project investigating medical phenomena of their interest.

10. To encourage students to reflect upon their design process and identify areas of strength and weakness in order to prepare for subsequent design exercises.

For this new version of the course, lecture and lab components have been blended into a weekly structure which includes two-hour instructional sessions on Monday and Wednesday followed by a one-hour session on Friday. The course, which is team-taught by two faculty in order to assure adequate technical instruction in each of the content areas, is broken down into four major three-week modules including biofluid mechanics, biomedical mass transport, bioinstrumentation and biomechanics. The final two weeks of the course are dedicated to the completion of student design projects which are designed to permit the students to utilize hands-on knowledge gained in lab to design, conduct and analyze open-ended experiments.

Facilities

In order to effectively integrate traditional lecture and laboratory components into single instructional sessions, we have designed and constructed a new instructional facility. This facility consists of two neighboring instructional rooms which are joined together by a smaller prep room as pictured in figure 1. The Hybrid Instructional Facility consists of a wet lab bench along the left-hand side, mobile chairs and tables which seat up to 20 students, full audio-visual equipment and two walls of dry erase boards. This room is connected to the Biotransport Laboratory via the Prep Room which contains lab supplies, tools, laptop computers and electronic equipment. The Biotransport Laboratory is designed as a full wet lab with

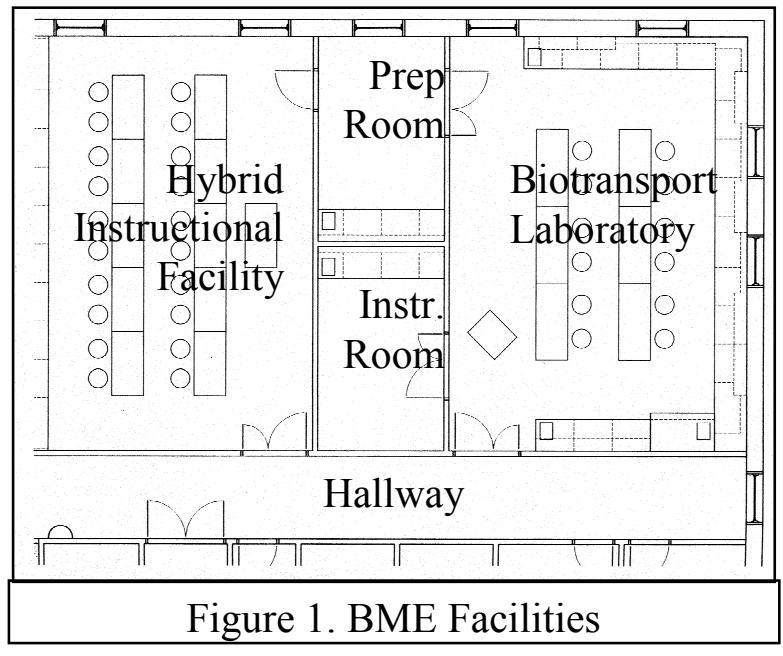
peripheral bench-tops, fume hood and mobile furniture. This room is equipped with white boards and overhead projection via a laptop. The primary functional purpose of these spaces is 
to allow classes to be taught while providing student and equipment mobility, instructional flexibility and easy access to a range of experimental needs. The primary pieces of equipment located in these facilities includes: syringe and peristaltic fluid pumps and necessary supplies; wireless laptop computers with data acquisition capabilities; BioPac physiological systems; CleveMed physiological systems; fluid characterization equipment; mass balances; pressure, temperature, conductivity, and $\mathrm{pH}$ measuring devices; laboratory microscopes; biomechanics force platform; function generators; power supplies; oscilloscopes. In general, the facilities have been outfitted to simultaneously run up to six stations of a wide range of experiments.

Integrated Instructional Sessions

As this introductory biomedical engineering course was broken into four content based modules, we present here two examples from three of the modules in order to demonstrate the delivery of the course. Due to course development limitations, the biomechanics module was not offered in the integrated fashion. As the class had two two-hour sessions each week and a single one-hour session, a majority of the integrated sessions occurred during the two hours periods. Although it was not followed exactly each time, a target class format was constructed as a guide to develop the class. This format is outlined below and shows how a 120 minute period was structured.

\begin{tabular}{ll} 
Time (min.) & Session Content \\
\hline $0-20$ & Introduction of concepts \\
$20-30$ & Example analytical problem \\
$30-40$ & Continuation of concept discussion \\
$40-60$ & In-class group problem solving session \\
$60-70$ & Introduction of hands-on exercise \\
$70-120$ & Hands-on exercise
\end{tabular}

Although this plan could not always be followed due to time consuming in-class problems or experiments, it provided a general guide to ensure the integration of the course components.

\section{Biofluid Mechanics}

For the area of biofluid mechanics, we present two examples of integrated instructional sessions. First, for providing students with an introduction to viscosity, the class began with a discussion of the physical origins of shear stresses followed by a derivation of Newton's Law of Viscosity and a discussion of Newtonian fluids. Next, the students broke into small groups to work on a problem requiring the utilization of Newton's Law of Viscosity. Following this in-class problem, non-Newtonian fluid properties were introduced and discussed. At the end of this lecture time, the students again broke into small groups to use a viscometer to measure viscosity as a function of shear rate for mineral oil (Newtonian) and shampoo (non-Newtonian). As a conclusion to this topic, the next lecture began with a discussion of blood rheology and Casson fluid behavior.

After presenting integrated sessions on fluid statics and Bernoulli's Equation without frictional losses, an integrated session focusing on Bernoulli's Equation including the frictional losses associated with tubing and tubing connections was conducted. Prior to this session, the students 
had already been introduced to the mechanical energy balance and the frictionless Bernoulli's equation through integrated sessions involving energy discussions and the utilization of BioPac systems to evaluate their own respiratory mechanics. In this next session, the instructor began with a physical discussion of the origin of friction and the relevance to pressure-flow relationships. Next, the Reynolds number was introduced followed by a discussion of the sources of frictional losses in a piping system. Before moving on to the hands-on lab portion, the students were assigned a group in-class problem which focused on analyzing the friction losses in a given piping network. For the hands-on lab portion of the session, the students constructed a recirculating flow system utilizing pumps, pressure gauges, tubing, fluid reservoirs and various fittings. By measuring the pressure drop in a specific section of the system, the students could compare the experimental and analytical frictional losses.

\section{Biomedical Mass Transport}

In the area of biomedical mass transport, students were presented with a number of integrated instructional sessions involving computer simulations, theoretical models and experiments. For example, in applying an unsteady mass balance to a recirculating flow system, the students were able to compare the results of their analytical methods to experimental data. In this session, students were taken through a derivation of an unsteady mass balance after being introduced to the concept of drug infusion. Next, an in-class problem was assigned where students needed to apply the unsteady mass balance to a problem where dye was being injected into a recirculating flow system. From their analytical results, students could predict the effects of changing variables such as infusion flow rate and concentration or the bulk flow rate. Following this analytical model development, the students broke into small groups and used their skills from the biofluid mechanics sessions to design a experiment that mimicked their model. In their experiments, students were able to monitor the concentration of dye in the recirculating system as a function of time using Vernier data acquisition systems. At the end of the experiments, students were able to compare and contrast the experimental and analytical results.

As a culminating experiment in biotransport, students were able to utilize a hemodialysis simulation unit in the laboratory to simulate actual hemodialysis. Following an introduction to renal physiology, the clinical aspects of dialysis and the associated transport mechanisms, groups of students carried out individual dialysis simulations. These experiments, which involved the transfer of salt from a salt solution, i.e. the blood, to a salt free solution, i.e., the dialysate, were designed to be unique to each group as the setups allow for a large variation in blood and dialysate flows and pressures. While the focus was on the overall mass transport, students could also utilize the various fluid mechanics concepts previously discussed in order to evaluate the shear stresses on the fluid in the tubing or the laminar or turbulent nature of the fluid flow.

\section{Bioinstrumentation}

In the bioinstrumentation module, a series of integrated sessions were created to convey the technical information and associated hands-on applications to the students. For example, one class session was utilized to introduce students to biological signals, namely the electrocardiogram (ECG). The session began with an introduction to cardiac physiology including action potential generation, the cardiac conduction pathway and the physiological 
significance of the components of the ECG. After this introduction, students were broken into groups where they used CleveMed physiological data acquisition systems to record their own ECGs. They examined alterations of the signals due to exercise, breathing, limb motion and $60 \mathrm{~Hz}$ noise. Following this hands-on exercise, the students gathered as a group to discuss various arrhythmias and their origin.

To provide the students with a more hands-on exposure to the actual data acquisition process, a series of hands-on exercises were developed to introduce the students to concepts ranging from signal components and aliasing to data acquisition and filtering. While the three experiments were presented in fall 2004 in a single lab session, we are planning to break them up into three instructional sessions for fall 2005 to conform to the target instructional session format presented earlier. The first of these three sessions will begin with a discussion of the basic components of a signal, i.e., amplitude and frequency, followed by an introduction to filtering and aliasing. An in-class problem emphasizing sampling frequencies and signal aliasing will precede a hands-on exercise where the students use various pieces of instrumentation to excite a pressure transducer and to sample output signals. The comparison of excitation signal characteristics with those of the output signal will allow the students to get a firmer grasp on concepts such as aliasing and appropriate sampling rates. For the second session, students will be presented with information regarding how to actually take data in a lab setting, i.e., how to use data acquisition cards and computers, how to perform the appropriate wiring with the instrumentation, and how to sample data appropriately. Following these discussions, students will be broken into small groups where they utilize previous knowledge to create a recirculating flow system with the new pressure transducers incorporated. With this system, students will be able to measure signal amplitudes to quantify pressure drops in addition to signal frequencies produced by the peristaltic pump. In the third and final session, students will be give more exposure to signal processing and filtering through lecture sessions and through the use of digital filters to filter the data they acquired with the recirculating systems. As a whole, these three sessions will provide the students with a intense and hands-on exposure to instrumentation and data acquisition.

Conclusions

Overall, the new version of our Introduction to Biomedical Engineering course offered in fall 2004 appears to have been successful in presenting the students with a course where the boundaries between lab and lecture are less distinct. Through the development of a number of integrated instructional sessions, the students received immediate reinforcement of technical concepts through the utilization of analytical, computer and experimental exercises.

In the written comments in the course evaluations, the students repeatedly expressed their satisfaction with the integrated format with comments such as "Most times, the class, despite being in a two hour block, wasn't boring because of the infusion of labs...." and "I liked the structure - having the lecture/lab integrated." When the students were asked about the value of the integration of the lecture and lab components in the biotransport modules, they gave an average score of 4.62 out of 5.0 further indicating their satisfaction with the format. A further indicator of the effectiveness of the integrated instructional sessions was apparent in the semester long projects. For these projects, which required students to utilize skills gained in the hands-on exercises throughout the semester, all of the groups of students designed and carried out 
experiments which utilized numerous skills from the integrated sessions. With their previously obtained understanding of the operation of the equipment, the students were able to focus on the more advanced and complicated aspects of their projects and not the basic operation of the equipment. This allowed the groups to achieve more in their projects and to design more complicated systems. In fact, the students commented that the mere fact that they knew they already had experience with a range of equipment permitted them to design more complicated systems and challenge themselves even more.

While the new format of this course has been well received by the students and appears to have permitted them to achieve more in their independent work in the course, we are aware of a number of challenges to offering this type of course. First, this new format required a significant investment of faculty time to create the integrated instructional sessions. However, once the sessions are designed, future modifications should ideally not require extensive time. The second challenge is in the area of equipment where this course format requires frequent set up and take down of multiple stations of identical equipment which can present staffing, space and equipment cost issues. Next, due to the need to accomplish set tasks in the sessions, the instructional plans can lack flexibility and be constrained by the timed class periods. One possible approach to remedy this issue in the future is to utilize the Friday one-hour session as a back-up or catch-up session for the integrated sessions run the previous Monday and Wednesday. Despite these challenges, we feel that the overall effectiveness of this style of teaching has benefited the students and even made the course more interesting and exciting for the faculty to teach.

In the future in our introductory course, we plan to continually modify the integrated structure of the instructional sessions in order to increase their effectiveness. Furthermore, we hope to incorporate more problem based learning or challenge based learning techniques into the course along with appropriate assessment methodologies. Overall, we feel that this first implementation of this instructional technique has been successful and we hope to optimize its use in this introductory course as well as introduce it into future biomedical engineering courses.

\section{Bibliography}

1. Beasley, C. A., Culkowski, P. M. and Guffner, G. E., Integration of Lecture and Laboratory in a Technology Program, Engineering Education, 433, April, 1990.

DANIEL P. CAVANAGH is an associate professor of biomedical and chemical engineering at Bucknell University in Lewisburg, PA. In addition to currently holding the Emmitt Memorial Chair in Biomedical Engineering, he is also Director of the Biomedical Engineering Program. Dr. Cavanagh teaches a variety of biomedical and chemical engineering courses in addition to researching intravascular gas embolism and dialysis.

SAMANTHA J. RICHERSON is an assistant professor of biomedical and electrical engineering at Bucknell University. Dr. Richerson teaches a range of biomedical and electrical engineering courses in addition to research that incorporates psychophysics into postural control testing in healthy and diabetic elders. She also researches theoretical models of neuronal signal transduction in the peripheral nervous system. 